\section{Hierarchical co-regulation}

Numerous studies have shown that long-range chromosomal contacts coincide with changes in gene expression. To analyze whether such contacts are required for cotranscription in multigene complexes that are activated by the same transcription factor, Mhlanga and colleagues studied a well-

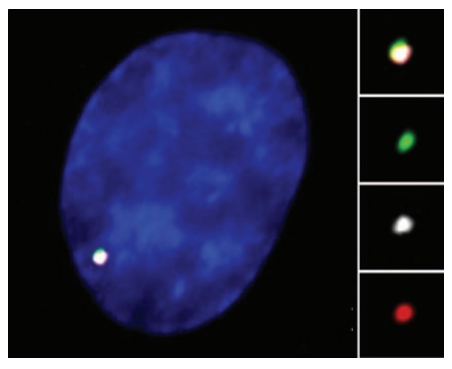
characterized tumor necrosis factor- $\alpha$ (TNF- $\alpha$ )-induced multigene complex, in which three interacting genes (SAMD4A, TNFAIP2 and SLC6A5) are activated by transcription factor NF- $\kappa B$. TNF- $\alpha$-induced formation of chromosomal contacts between these genes was accompanied by cotranscription of all three genes in a relatively small fraction of cells, thus suggesting that only certain cells in a population have the correct spatial arrangement of their chromosomes to permit coregulation. The authors therefore devised a single-cell-based assay, in which TALE nuclease (TALEN)-mediated disruption of the SAMD4A gene loop in single cells was visualized by DNA fluorescence in situ hybridization (FISH), and abrogated SAMD4A RNA and protein expression was analyzed by RNA FISH and immunofluorescence. Parallel monitoring of the other two interacting genes showed that their transcription was abolished. TALENs were also targeted to sites of chromosomal contacts in TNFAIP2 and SLC6A5 while transcription of the other two interacting genes was monitored. Interestingly, disruption of the TNFAIP2 loop did not have a significant effect on $S A M D 4 A$ transcription, yet transcription of both TNFAIP2 and SLC6A5 was reduced, thus suggesting a hierarchical mode of regulation. Disruption of the SLC6A5 loop had an even greater hierarchical effect, as transcription of both interacting genes was unaffected. Disrupted gene loops could be successfully repaired in a sequence-independent manner to restore transcription of the interacting genes. The single-cell TALEN assay seems a powerful approach to interrogate transcriptional regulation in three dimensions, and it will be interesting to explore the molecular basis for the hierarchical mode of transcription in a multigene complex. (Cell 155, 606-620, 2013)

$A H$

\section{Found in translation}

The immune system differentiates self from nonself through the display of antigens on the cellular surface in association with class I major histocompatibility complex (MHC) molecules. Although the basic principle of immune peptide presentation by the MHC class I system is well understood, the exact provenance of the peptides used in this pathway remains poorly defined. Building on their previous work demonstrating that the pioneer round of mRNA translation is a major source of antigenic peptide substrates, Fåhraeus and colleagues now show that prespliced RNAs - translated in the nucleus through a noncanonical mechanism-can be a source of peptides for the MHC class I pathway. To detect early ribosomal scanning events that give rise to antigenic peptide products, the authors engineered constructs for expression of exogenous antigenic peptides from intronic sequences within the $\beta$-globin gene and confirmed that the mRNAs' presence within the cell was limited to the nuclear compartment. The presentation of the antigenic peptides was reduced by $\sim 100$ fold when their gene sequences were placed in a reading frame different from that of the host gene, thus indicating that the peptides are the translation products of unspliced mRNAs. Because the unspliced reporter mRNA was restricted to the nucleus, the authors surmised that the synthesis of pioneer translation products for the MHC class I pathway must occur within this compartment. Accordingly, forcing export of the unspliced construct by fusion with the HIV REV element reduced antigen presentation. Finally, the authors could detect the colocalization of nascent peptide products and ribosomal proteins within the nucleus, confirming active nuclear translation. Although the existence and possible function of nuclear translation have been controversial, the new findings described here provide evidence for nuclear ribosomes being part of a specialized translation system that is key to immune surveillance. (Proc. Natl. Acad. Sci. USA 110, 17951-17956, 2013)

\section{Living without origins}

DNA replication origins are the sites of assembly of replicationfactor complexes that duplicate the genome, and they direct initiation in cells from all domains of life. Whereas bacterial chromosomes are replicated from a single origin, eukaryotes and most archaea use multiple origins and share similar replication machinery. Recent work by Allers and colleagues using the model halophile Haloferax volcanii suggests that this archaeal genome can also be replicated by an originindependent mechanism. The circular main chromosome of wild-type $H$. volcanii has three origins (oris) that have different replication efficiencies. The replication profile of the $H$. volcanii laboratory strain showed a different pattern of ori utilization, which subsequent mapping revealed was due to chromosomal integration of the megaplasmid pHV4, resulting in four genomic oris. Remarkably, deletion of each ori singly and in combination to assess their relative activities revealed that cells lacking all four oris were still viable and had the same DNA content as did cells with a full complement of origins. Moreover, cells with no oris exhibited faster growth rates than did cells with all oris intact. Mapping of replication initiation sites in the ori-deleted strain identified no new discrete peaks in DNA copy number, which would have reflected activation of dormant origins. Instead, the orideleted strain showed a flat replication profile characteristic of dispersed initiation events. Genetic deletion analysis demonstrated that the ori-deleted cells are dependent on the $\operatorname{rad} A$ gene encoding the archaeal homolog of bacterial RecA recombinase. Like RecA, RadA is essential for homologous recombination. Previous work has shown that Escherichia coli can use homologous recombination to initiate replication in the absence of a functional ori, but this mechanism severely compromises cell growth. The current work provides the first example of efficient duplication of an entire chromosome through recombination-dependent replication and raises intriguing questions concerning the efficiency and advantage of origin-directed replication to be addressed by future studies. (Nature doi:10.1038/nature12650, 3 November 2013) BM 